\title{
Human Development Equally Important For Economic Development
}

\author{
${ }^{1}$ Dr. RK Arya, ${ }^{2}$ Dr. Urvashi Sharma, ${ }^{3}$ Dr. Arvind Dalal, \\ ${ }^{1}$ Professor of Economics and Chairman, Doctoral Research Board, MRIIIRS, FARIDABAD \\ ${ }^{2}$ Assistant Professor, FCBS, MRIIIRS, Faridabad \\ ${ }^{3}$ Assistant Professor, FCBS, MRIIRS, Faridabad
}

Article History: Received: 11 January 2021; Accepted: 27 February 2021; Published online: 5 April 2021

\begin{abstract}
Many States are failing to keep the momentum up in pushing the growth rate either because their social capacity is not up to the brim and people are struggling to get two ends meal. What the States are needed to keep continuing with investment at ever increasing rate and deal social priorities differently? There are many States which face the wrath of anti-incumbency every five years the reason for this larger section of the people remain untouched with the development schemes. It is their bad luck that neither did they get the benefit of moving up in the social hierarchy nor they are covered with some employment guarantee scheme. The vulnerability during the pandemic have further added the burden of making the people socially strong. Education and health both have gone through serious setback.
\end{abstract}

There are two ways to look Indian economy not growing at proper pace good enough to keep improving the per capita income and pulling up the people above the poverty line. The one is the ratio of revenue and capital expenditure where more revenue is diverted to subsidies and wages to the public servant. The other is that development expenditure is significantly attractive. While revenue expenditure is meant for consumption and translates into one demand, the development expenditure is more biased towards human and material development. The developed countries by definition are so because they have crossed over to the material and human development even though at one point in time these were not so good.

The developing countries also lack resources to start moving towards development the same way when the developed countries also had not resources good enough to move to higher level. Indian situation at the time of independence was deplorable because British rule extended over a period of 200 years was only to use the resources to be deployed for their power building. India was expected to lay down the model of development in which the importance was given to creation of public establishments as far as possible. This was done with a view because each person could be given equal attention in terms of their needs. While the idea of development was same, India started facing an alarming situation whereby poverty was acutely high to extent socialistic leaders attempted to shake the government through protests in 1970s. The failure of the public sector to maintain social order was visibly under question but the condition did not permit to alter the system of development. Public sector gives way to leakages several authors have pointed out. There is no perception of productivity in the public sector as well because every employee there has to work for limited hours without any pressure from the boss.

Public sector was not capable of delivering the expected fruits evident from the large number of public sector companies becoming sick and running into loss which in the midst had to be closed down and very easily replaced by the other convenient compositions not harsh to the public i.e. co-operative sector. The model of development adopted in 1950s came under severe criticism in 1990s when the government was under duress and was absolutely difficult to run the course in the manner it was being run for long time. Without any further option, some change in the model was decided and it was to give some place to private sector as well which was prohibited or allowed on case to case basis. But before the situation became alarming, some of the States within the country were identified to be backward and hardly progressing particularly, Bihar, Madhya Pradesh, Rajasthan and Uttar Pradesh. Chhattisgarh was part of Madhya Pradesh till then. Since 1960s, each State was getting their share from the centre besides they are expected to make their own revenue out of the taxes.

Agriculture is exempted from taxes ever as before and now. Agriculture was a good source of income to the people of all States but the difference lied in making the progress using an appropriate strategy. Since agriculture contributed $45-50 \%$ in GDP on all India basis, some space was there for secondary and tertiary sector as well. It is matter of pushing the wheels of progress. One way is to focus on human development so that all the sectors could be expanded as much as possible. The second way is to focus on maintaining the social order and keep working for the welfare of the people besides mal-governance in which leakages are obvious. As the States were same any time and these were given equal treatment before Independence but the question arises how do the States remained backward and other have moved far ahead. This point needs to be investigated based on the limited data available. 
The pattern of utilization of monetary resources and availability of natural and easy resources would make the point still more clear. Considering the leveled status of development across States some States stand tall and other are still struggling with their odds. Differences on account of caste and natural impact even though cannot be easily ignored but there are still ways to develop with the feeling of competition. The migration from one State to other in huge number present a critical view about the States not coming up so well. Migration in search of work in other States to continue to live with nominal standard does not find any appreciation. However, migration for the sake of well-paid jobs is praiseworthy. In migration to other States, it is difficult to get all amenities which are available to the residents of that State. Therefore, further scope of development is obviously stunted. Children are unable to eat good food and cannot go to schools where some minimum aspirations are also fulfilled. While father continues to be a casual worker what are the chances the son will grow up to earn handsomely because either it is the educational background or the wealth effect play their roles in making the destiny of any person. Small probability to the chance factor cannot however, be ruled out. The literacy rate, employment rate, poverty ratio and distribution of GDP across sectors no doubt are the positive factors which explain the progress of any State but nonetheless how far these are made possible are also important. Whether development has paced without focused approach or it has been allowed with systematic efforts would be revealed from the pattern of expenditure i.e., infrastructure, education, health or merely subsidies. Some data which is available from the published sources is worth examining. While the government at the centre makes projection of growth almost every year, but there are acute slippages from what is being projected. It makes revenue forecast unrealistic and fiscal deficit also spins out of control. States during the last three years are also complaining that they are not adequately compensated by the centre and hence the development is hazy. Some States have not succeeding the vulnerability of the people and their issues about the healthy life always attract political attention which is being converted into freebies and doles or if not then poverty continues to persist.

In a State where poverty is above average these States are also performing. Their revenue expenditure is skewed in running the schemes for poor. Some States which somehow had gone faster with the development agenda, their development is really focused on human development. The employment, stabilizing population or at least putting a check on the growth of population is their priority. This has been happening because of the increased expenditure on education and health besides the increased focus on women employment and training is also considered as one of the factor giving them the advantage of growth. decadal growth indicates that top 5 States are endeavoring to stabilize the population by spending more on education and health. The progressive States have their capital expenditure slightly in the increasing order. In India it is difficult for the moment to get the capital expenditure around $20 \%$ of the budget because the States have more commitment to meet the revenue expenditure to keep generating the wage based or casual employment. Billion of Rupees are given to farmers and reserved for health delivery at tertiary level and hence to satisfy the needy section of the society. There is a problem of credit delivery system. Large companies consuming lions share and therefore their growth is not enough to make an assessment of the MSME sector which keeps struggling with the sustainability. Apart from the poor production and logistics conditions prevailing there, the MSMEs struggle with the finances cannot be underestimated. Government in the last few days succeeded in addressing the employment issues of the rural areas by creating pan India employment guarantee program but there is still acute tendency of the labor force from weak avenue States to move to cities in some other States where they are managing to take casual or half baked jobs. There is no estimate available whether they are crossing the poverty line on permanent basis or they can slip to poverty any time. These people are remitting the some funds in their homes but these could be considered as supplementary and it is the income earned by the rest of family income which should be good enough to decide whether these families are living above poverty line.

The States which have made efforts in increasing productivity in agriculture, their surplus labor force from the rural areas is absorbed in the services and industries in the rural and urban areas. The rate of urbanization is directly related to productivity in the agriculture sector. Punjab and Haryana have gone far ahead in increasing the productivity in the agriculture sector and hence their employment rate is also satisfactory. During the last two decades, Gujarat has also made efforts to strike balance in the sect oral composition of the growth and employment. The Indian macro economic growth though to some extent dependent on the States but centre is also playing huge role in designing the policies for the growth in the States. Literacy rate is an indicator of the developed States and it is higher in high growth States. The decadal population growth rate, technical institutions and literacy which is the proxy for controlled education environment are considered good for delivering the best in terms of economic growth. The States which have moderate to high revenue deficit are facing shortage in durable funds to be used in creating social infrastructure and physical infrastructure. The centre government is nudging all the States to maintain cleanliness and also provide tap water across the length and breadth of the country. Neutralizing the revenue deficit 
${ }^{1}$ Dr. RK Arya, ${ }^{2}$ Dr. Urvashi Sharma, ${ }^{3}$ Dr. Arvind Dalal,

depends upon the States own earning and weaker States have less to claim for taxes because tax in the States are again dependent on the development.

India Literacy Rate: State Wise Report

\section{Literacy rates across the States}

\begin{tabular}{|l|l|}
\hline \multicolumn{1}{|c|}{ State } & $\begin{array}{c}\text { Overall Literacy Rate } \\
\text { Urban + Rural) (Male \& Female) }\end{array}$ \\
\hline Andhra Pradesh & 66.4 \\
\hline Assam & 85.9 \\
\hline Bihar & 70.9 \\
\hline Chhattisgarh & 77.3 \\
\hline Delhi & 88.7 \\
\hline Gujarat & 82.4 \\
\hline Haryana & 80.4 \\
\hline Himachal Pradesh & 86.6 \\
\hline Jammu \& Kashmir & 77.3 \\
\hline Jharkhand & 74.3 \\
\hline Karnataka & 77.2 \\
\hline Kerala & 96.2 \\
\hline Madhya Pradesh & 73.7 \\
\hline Maharashtra & 84.8 \\
\hline Odisha & 77.3 \\
\hline Punjab & 83.7 \\
\hline Rajasthan & 69.7 \\
\hline Tamil Nadu & 82.9 \\
\hline Telangana & 72.8 \\
\hline Uttarakhand & 87.6 \\
\hline Uttar Pradesh & 73.0 \\
\hline West Bengal & 80.5 \\
\hline All India & 77.7 \\
\hline Overall, the & 59.7 \\
\hline
\end{tabular}

Overall, the male literacy rate in the country is much higher at $84.7 \%$ as compared to female literacy rate of $70.3 \%$.

The literacy rates in urban population was better at $87.7 \%$ overall as compared to rural region at $73.5 \%$. Even in urban areas, however, female literacy lagged behind male literacy rate

\section{State-wise GDP of the States of India}

List of Indian states and union territories by GDP 
Human Development Equally Important For Economic Development

State-wise GDP of the States of India

List of Indian states and union territories by GDP

\begin{tabular}{|c|c|c|c|}
\hline Rank & State/Union Territory & $\begin{array}{c}\text { Nominal GDP } \\
\text { ₹ lakh crore = INR trillions; } \\
\text { USD billions }\end{array}$ & Data year \\
\hline 1 & Maharashtra & ₹28.78 lakh crore (US $\$ 400$ billion) & $2019-20$ \\
\hline 2 & Tamil Nadu & ₹18.46 lakh crore (US $\$ 260$ billion) & $2019-20$ \\
\hline 3 & Uttar Pradesh & ₹17.94 lakh crore (US $\$ 250$ billion) & $2019-20$ \\
\hline 4 & Karnataka & ₹16.99 lakh crore (US $\$ 240$ billion) & $2019-20$ \\
\hline 5 & Gujarat & ₹15.03 lakh crore (US $\$ 210$ billion) & $2018-19$ \\
\hline 6 & West Bengal & ₹12.54 lakh crore (US $\$ 180$ billion) & 2019-20 \\
\hline 7 & Rajasthan & ₹10.21 lakh crore (US $\$ 140$ billion) & $2019-20$ \\
\hline 8 & Andhra Pradesh & ₹9.73 lakh crore (US $\$ 140$ billion) & $2019-20$ \\
\hline 9 & Telangana & ₹9.69 lakh crore (US\$140 billion) & $2019-20$ \\
\hline 10 & Madhya Pradesh & ₹9.09 lakh crore (US\$130 billion) & $2019-20$ \\
\hline 11 & Delhi & ₹8.56 lakh crore (US $\$ 120$ billion) & $2019-20$ \\
\hline 12 & Haryana & ₹8.31 lakh crore (US $\$ 120$ billion) & $2019-20$ \\
\hline 13 & Kerala & ₹7.82 lakh crore (US $\$ 110$ billion) & $2018-19$ \\
\hline 14 & Bihar & ₹6.12 lakh crore (US\$86 billion) & $2019-20$ \\
\hline 15 & Punjab & ₹5.75 lakh crore (US $\$ 81$ billion) & 2019-20 \\
\hline 16 & Odisha & ₹5.31 lakh crore (US\$74 billion) & $2019-20$ \\
\hline 17 & Chhattisgarh & ₹3.29 lakh crore (US $\$ 46$ billion) & $2019-20$ \\
\hline 18 & Jharkhand & ₹3.29 lakh crore (US $\$ 46$ billion) & 2019-20 \\
\hline 19 & Assam & ₹3.16 lakh crore (US $\$ 44$ billion) & $2018-19$ \\
\hline 20 & Uttarakhand & ₹2.46 lakh crore (US\$34 billion) & $2018-19$ \\
\hline 21 & Himachal Pradesh & ₹1.65 lakh crore (US $\$ 23$ billion) & 2019-20 \\
\hline 22 & Jammu and Kashmir & ₹1.56 lakh crore (US $\$ 22$ billion) & $2018-19$ \\
\hline 23 & Goa & ₹0.731 lakh crore (US $\$ 10$ billion) & $2019-20$ \\
\hline 24 & Tripura & ₹0.461 lakh crore (US $\$ 6.5$ billion) & 2019-20 \\
\hline 25 & Chandigarh & ₹0.421 lakh crore (US $\$ 5.9$ billion) & 2018-19 \\
\hline 26 & Puducherry & ₹0.408 lakh crore (US\$5.7 billion) & $2019-20$ \\
\hline 27 & Meghalaya & ₹0.366 lakh crore (US\$5.1 billion) & 2019-20 \\
\hline 28 & Sikkim & ₹0.325 lakh crore (US $\$ 4.6$ billion) & $2019-20$ \\
\hline 29 & Manipur & ₹0.279 lakh crore (US\$3.9 billion) & 2018-19 \\
\hline 30 & Arunachal Pradesh & ₹0.246 lakh crore (US $\$ 3.4$ billion) & $2018-19$ \\
\hline 31 & Nagaland & ₹0.273 lakh crore (US $\$ 3.8$ billion) & $2018-19$ \\
\hline 32 & Mizoram & ₹0.195 lakh crore (US $\$ 2.7$ billion) & $2018-19$ \\
\hline 33 & Andaman and Nicobar Islands & ₹0.079 lakh crore (US\$1.1 billion) & $2017-18$ \\
\hline
\end{tabular}

State-wise Distribution of GDP across the sectors

Distribution of gross domestic product (GDP) across economic sectors in India 2019

In 2019, almost half of India's GDP was generated by the services sector, a slight and steady increase over the last 10 years. Among the leading services industries in the country are telecommunications, IT, and software.

\section{The IT factor}

The IT industry is a vital part of India's economy, and in the fiscal year of 2016/2017, it generated about 8 percent of India's GDP alone - a slight decrease from previous years, when it made up about 10 percent of the country's economy. Nevertheless, the IT industry is growing, as is evident by its quickly increasing revenue and employment figures. IT includes software development, consulting, software management, and online services, and business process management $(\mathrm{BPM})$. 


\section{Employee migration}

Although employment figures in IT, and thus in the services sector, are on the rise, most of the Indian workforce is still employed in agriculture, however, the figures show a trend pointing towards a reversal of this distribution. For now, the majority of Indians still do not live in cities - where IT jobs are generated - but urbanization is on the rise as well.

India: Distribution of gross domestic product (GDP) across economic sectors from 2009 to 2019

\section{India: Distribution of gross domestic product (GD sectors from 2009 to 2019}

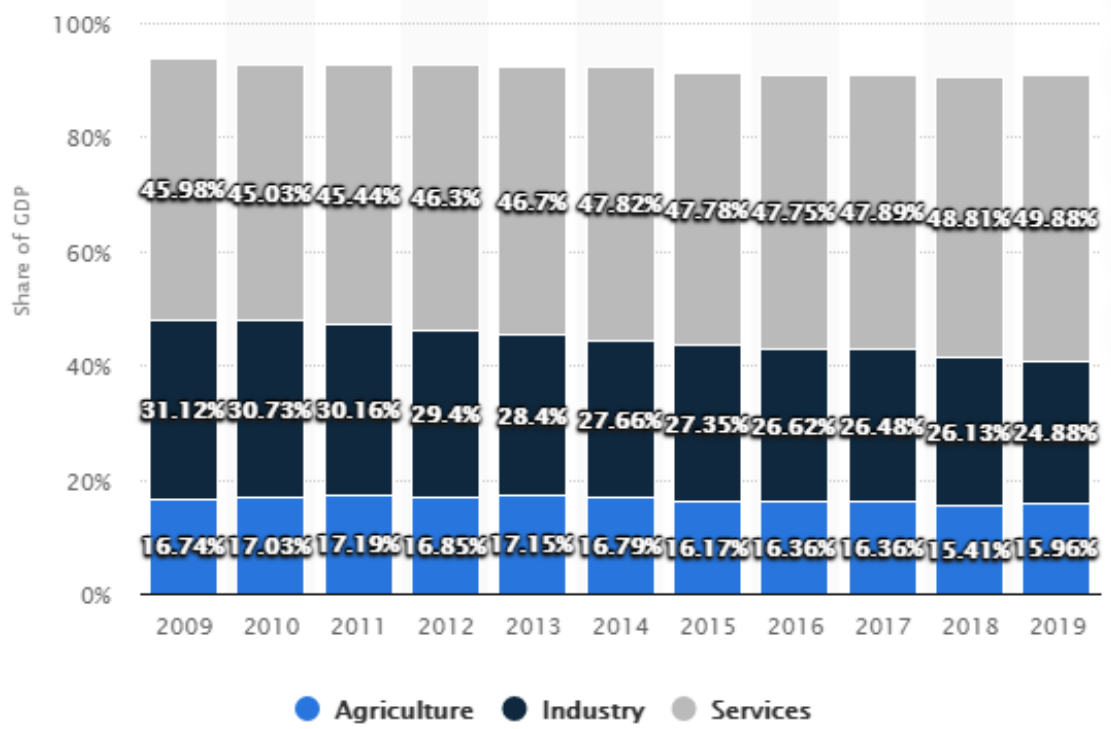

State-wise poverty ratio

\begin{tabular}{|c|c|c|}
\hline Rank & State/U.T. & $\begin{array}{c}\text { Poverty (\% of people } \\
\text { below poverty line) }{ }^{[\mathbf{3}]}\end{array}$ \\
\hline 1 & Goa & 5.09 \\
\hline 2 & Kerala & 7.05 \\
\hline 3 & Himachal Pradesh & 8.06 \\
\hline 4 & Sikkim & 8.19 \\
\hline 5 & Punjab & 8.26 \\
\hline 6 & Andhra Pradesh & 9.20 \\
\hline 7 & Haryana & 11.16 \\
\hline 8 & Uttarakhand & 11.26 \\
\hline 9 & Tamil Nadu & 11.28 \\
\hline 10 & Meghalaya & 11.87 \\
\hline 11 & Tripura & 14.05 \\
\hline
\end{tabular}




\begin{tabular}{|c|c|c|}
\hline 12 & Rajasthan & 14.71 \\
\hline 13 & Gujarat & 16.63 \\
\hline 14 & Maharashtra & 18.35 \\
\hline 15 & Nagaland & 19.98 \\
\hline 16 & West Bengal & 20.4 \\
\hline 17 & Mizoram & 20.91 \\
\hline 18 & Karnataka & 21.92 \\
\hline$* *$ & All India Average & 29.43 \\
\hline 19 & Uttar Pradesh & 31.65 \\
\hline 20 & Madhya Pradesh & 31.98 \\
\hline 21 & Assam & 32.59 \\
\hline 22 & Odisha & 90 \\
\hline 23 & Bihar & 34.67 \\
\hline 24 & Arunachal Pradesh & 36.89 \\
\hline 25 & Manipur & 36.96 \\
\hline 26 & Jharkhand & 39.93 \\
\hline 27 & Chhattisgarh & N/A \\
\hline 28 & Telangana & 10.35 \\
\hline U/T & Lakshadweep & 2.77 \\
\hline U/T & Puducherry & 1 \\
\hline U/T & Daman and Diu & 9.69 \\
\hline U/T & Delhi & 9.86 \\
\hline U/T & Chandigarh & 9.91 \\
\hline NCT & Dadra and Nagar Haveli & 21.81 \\
\hline U/T & & 39.31 \\
\hline U/T & & \\
\hline & Andaman and Nicobar Islands & \\
\hline
\end{tabular}

State-wise percentage of Agriculture income in India

\begin{tabular}{|l|c|c|c|c|c|}
\hline Year & $\begin{array}{c}\text { Agriculture, forestry \& } \\
\text { fishing }\end{array}$ & Crops & Livestock & $\begin{array}{c}\text { forestry } \\
\text { and logging }\end{array}$ & $\begin{array}{c}\text { fishing and } \\
\text { aquaculture }\end{array}$ \\
\hline $2011-12$ & 18.5 & 12.1 & 4.0 & 1.5 & 0.8 \\
\hline $2012-13$ & 18.2 & 11.8 & 4.0 & 1.5 & 0.9 \\
\hline $2013-14$ & 18.6 & 12.1 & 4.1 & 1.5 & 0.9 \\
\hline $2014-15$ & 18.2 & 11.2 & 4.4 & 1.5 & 1.0 \\
\hline $2015-16$ & 17.7 & 10.6 & 4.6 & 1.5 & 1.1 \\
\hline $2016-17$ & 18.0 & 10.6 & 4.8 & 1.5 & 1.1 \\
\hline $2017-18$ & 18.3 & 10.5 & 5.1 & 1.4 & 1.2 \\
\hline $2018-19$ & 17.6 & 9.7 & 5.1 & 1.5 & 1.2 \\
\hline $2019-20$ & 18.4 & 10.7 & 5.2 & 1.3 & 1.2 \\
\hline $2020-$ & 20.2 & & & & \\
\hline 21 & & & & & 1.2 \\
\hline
\end{tabular}


${ }^{1}$ Dr. RK Arya, ${ }^{2}$ Dr. Urvashi Sharma, ${ }^{3}$ Dr. Arvind Dalal,

\section{State-wise number of technical institutions}

\section{AREA \& POPULATION}

\begin{tabular}{|c|c|c|}
\hline S.No & State/Union Territory & $\begin{array}{l}\text { Number of Engineering } \\
\text { Institutes }\end{array}$ \\
\hline 1 & Andaman \& Nicobar Islands & 1 \\
\hline 2 & Andhra Pradesh & 149 \\
\hline 3 & Arunachal Pradesh & 24 \\
\hline 4 & Assam & 37 \\
\hline 5 & Bihar & 107 \\
\hline 6 & Chandigarh & 14 \\
\hline 7 & Chhattisgarh & 73 \\
\hline 8 & Dadra and Nagar Haveli & 3 \\
\hline 9 & Daman and Diu & 2 \\
\hline 10 & Delhi & 56 \\
\hline 11 & Goa & 17 \\
\hline 12 & Gujarat & 197 \\
\hline 13 & Haryana & 226 \\
\hline 14 & Himachal Pradesh & 64 \\
\hline 15 & Jammu \& Kashmir & 53 \\
\hline 16 & Jharkhand & 73 \\
\hline 17 & Karnataka & 157 \\
\hline 18 & Kerala & 129 \\
\hline 19 & Madhya Pradesh & 241 \\
\hline 20 & Maharashtra & 334 \\
\hline 21 & Manipur & 4 \\
\hline 22 & Meghalaya & 7 \\
\hline 23 & Mizoram & 4 \\
\hline 24 & Nagaland & 8 \\
\hline 25 & Odisha & 242 \\
\hline 26 & Puducherry & 29 \\
\hline 27 & Punjab & 302 \\
\hline 28 & Rajasthan & 281 \\
\hline 29 & Sikkim & 5 \\
\hline 30 & Tamil Nadu & 314 \\
\hline 31 & Telangana & 253 \\
\hline 32 & Tripura & 13 \\
\hline 33 & Uttar Pradesh & 574 \\
\hline 34 & Uttarakhand & 117 \\
\hline 35 & West Bengal & 172 \\
\hline- & Total & 4282 \\
\hline
\end{tabular}

State-wise capital and revenue expenditure

Growth rate of expenditure and revenue receipts of states during the 2015-20 period

Note: Expenditure excludes debt repayment, and loans and advances given by the states.

Sources: State Budget Documents; PRS. 


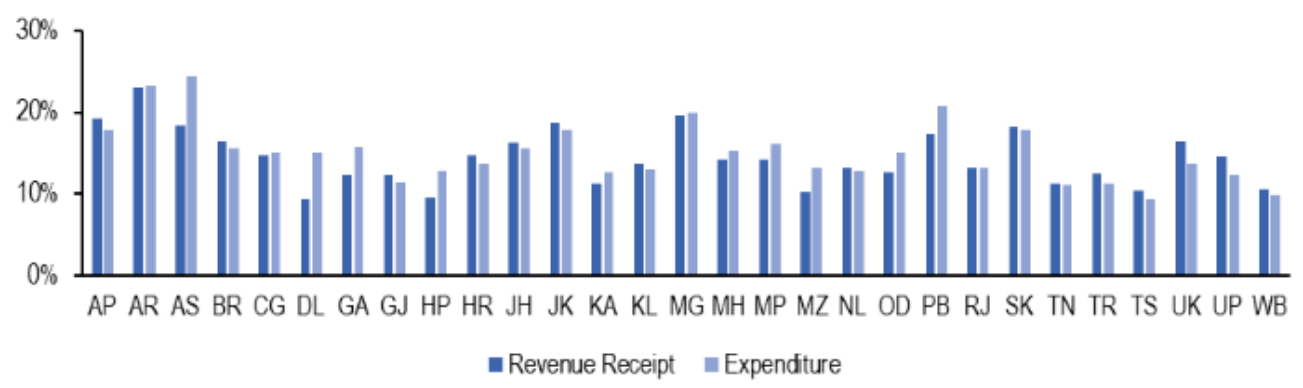

State -wise Percentage of expenditure spent on education, health and training sectors mave uecinleu.

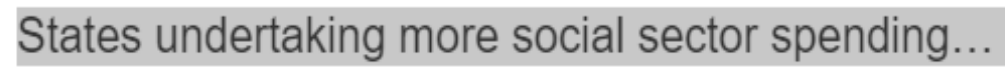
Total social sector spending by states (\% of GDP)

Education ${ }^{*}$ Health ${ }^{* *}$ Other social sector spending

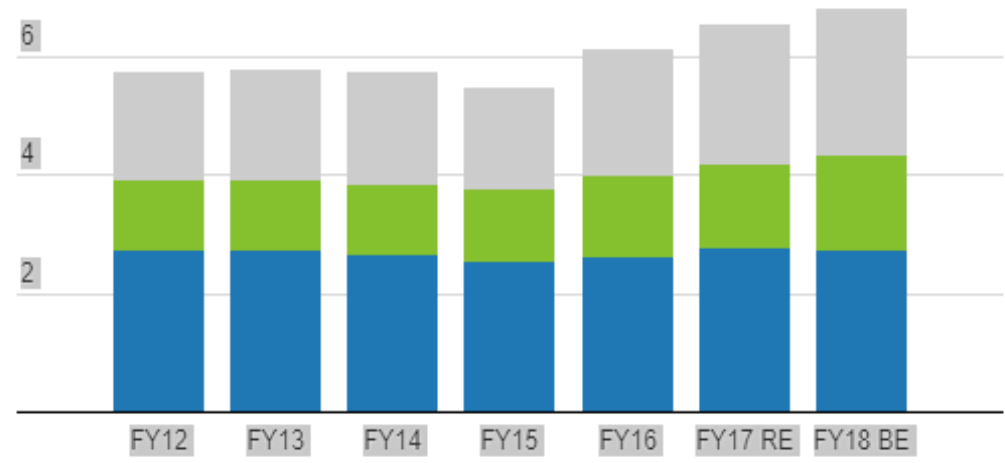

State-wise un employment situation across various sectors

\begin{tabular}{|l|l|}
\hline State or union territory & Unemployment rate $\mathbf{( \% )}$ \\
\hline Andhra Pradesh & 3.9 \\
\hline Assam & 1.6 \\
\hline Bihar & 11.5 \\
\hline Chhattisgarh & 6.2 \\
\hline Delhi & 8.0 \\
\hline Goa & 21.1 \\
\hline Gujarat & 3.2 \\
\hline Haryana & 26.4 \\
\hline Himachal Pradesh & 15.1 \\
\hline Jammu and Kashmir & 14.2 \\
\hline
\end{tabular}


${ }^{1}$ Dr. RK Arya, ${ }^{2}$ Dr. Urvashi Sharma, ${ }^{3}$ Dr. Arvind Dalal,

\begin{tabular}{|l|l|}
\hline Jharkhand & 12.1 \\
\hline Karnataka & 2.5 \\
\hline Kerala & 4.3 \\
\hline Madhya Pradesh & 2.1 \\
\hline Maharashtra & 3.8 \\
\hline Meghalaya & 3.8 \\
\hline Odisha & 2.5 \\
\hline Puducherry & 5.8 \\
\hline Punjab & 7.2 \\
\hline Rajasthan & 25.6 \\
\hline Sikkim & 4.3 \\
\hline Tamil Nadu & 4.8 \\
\hline Telangana & 5.7 \\
\hline Tripura & 11.1 \\
\hline Uttar Pradesh & 4.1 \\
\hline Uttarakhand & 4.7 \\
\hline West Bengal & 6.2 \\
\hline & \\
\hline
\end{tabular}

STATE WISE DECADAL GROWTH POPULATIONDate : Oct 13, 2020

Table 4: State-wise Decadal Growth Rate of Population

\begin{tabular}{|l|r|r|r|r|r|r|}
\hline \multicolumn{1}{|c|}{ State/Union Territory } & \multicolumn{1}{|c|}{$\mathbf{1 9 5 1 -}$} & $\mathbf{1 9 6 1 -}$ & $\mathbf{1 9 7 1 -}$ & $\mathbf{1 9 8 1 -}$ & $\mathbf{1 9 9 1 -}$ & \multicolumn{2}{|c|}{$\mathbf{2 0 0 1 -}$} \\
\hline Andaman and Nicobar Islands & 105.19 & $\mathbf{1 9 7 1}$ & $\mathbf{1 9 8 1}$ & $\mathbf{1 9 9 1}$ & $\mathbf{2 0 0 1}$ & $\mathbf{2 0 1 1}$ \\
\hline Andhra Pradesh & 15.65 & 20.90 & 23.10 & 24.20 & 14.59 & 10.98 \\
\hline Arunachal Pradesh & - & 38.91 & 35.15 & 36.83 & 27.00 & 26.03 \\
\hline Assam & 34.98 & 34.95 & 23.36 & 24.24 & 18.92 & 17.07 \\
\hline Bihar & 19.79 & 20.91 & 24.16 & 23.38 & 28.62 & 25.40 \\
\hline Chandigarh & 394.13 & 114.59 & 75.55 & 42.16 & 40.28 & 17.09 \\
\hline Chhattisgarh & 22.77 & 27.12 & 20.39 & 25.73 & 18.27 & 22.61 \\
\hline Dadra and Nagar Haveli & 39.56 & 27.96 & 39.78 & 33.57 & 59.22 & 55.88 \\
\hline Daman and Diu & -24.56 & 70.85 & 26.07 & 28.62 & 55.73 & 53.76 \\
\hline NCT of Delhi & 52.44 & 52.93 & 53.00 & 51.45 & 47.02 & 21.21 \\
\hline Goa & 7.77 & 34.77 & 26.74 & 16.08 & 15.21 & 8.23 \\
\hline Gujarat & 26.88 & 29.39 & 27.67 & 21.19 & 22.66 & 19.28 \\
\hline Haryana & 33.79 & 32.22 & 28.75 & 27.41 & 28.43 & 19.90 \\
\hline Himachal Pradesh & 17.87 & 23.04 & 23.71 & 20.79 & 17.54 & 12.94 \\
\hline Jammu and Kashmir & 9.44 & 29.65 & 29.69 & 30.89 & 29.43 & 23.64 \\
\hline Jharkhand & 19.69 & 22.58 & 23.79 & 24.03 & 23.36 & 22.42 \\
\hline
\end{tabular}


Human Development Equally Important For Economic Development

\begin{tabular}{|c|c|c|c|c|c|c|}
\hline Karnataka & 21.57 & 24.22 & 26.75 & 21.12 & 17.51 & 15.60 \\
\hline Kerala & 24.76 & 26.29 & 19.24 & 14.32 & 9.43 & 4.91 \\
\hline Lakshadweep & 14.61 & 31.95 & 26.53 & 28.47 & 17.30 & 6.30 \\
\hline Madhya Pradesh & 24.73 & 29.28 & 27.16 & 27.24 & 24.26 & 20.35 \\
\hline Maharashtra & 23.60 & 27.45 & 24.54 & 25.73 & 22.73 & 15.99 \\
\hline Manipur & 35.04 & 37.53 & 32.46 & 29.29 & 24.86 & 24.50 \\
\hline Meghalaya & 27.03 & 31.50 & 32.04 & 32.86 & 30.65 & 29.95 \\
\hline Mizoram & 35.61 & 24.93 & 48.55 & 39.70 & 28.82 & 23.48 \\
\hline Nagaland & 73.20 & 39.88 & 50.05 & 56.08 & 64.53 & -0.58 \\
\hline Odisha & 19.82 & 25.05 & 20.17 & 20.06 & 16.25 & 14.05 \\
\hline Puducherry & 16.34 & 27.81 & 28.15 & 33.64 & 20.62 & 28.08 \\
\hline Punjab & 21.56 & 21.70 & 23.89 & 20.81 & 20.10 & 13.89 \\
\hline Rajasthan & 26.20 & 27.83 & 32.97 & 28.44 & 28.41 & 21.31 \\
\hline Sikkim & 17.76 & 29.38 & 50.77 & 28.47 & 33.06 & 12.89 \\
\hline Tamil Nadu & 11.85 & 22.30 & 17.50 & 15.39 & 11.72 & 15.61 \\
\hline Tripura & 78.71 & 36.28 & 31.92 & 34.30 & 16.03 & 14.84 \\
\hline Uttar Pradesh & 16.38 & 19.54 & 25.39 & 25.61 & 25.85 & 20.23 \\
\hline Uttarakhand & 22.57 & 24.42 & 27.45 & 23.13 & 20.41 & 18.81 \\
\hline West Bengal & 32.80 & 26.87 & 23.17 & 24.73 & 17.77 & 13.84 \\
\hline ALL INDIA & 21.51 & 24.80 & 24.66 & 23.87 & 21.54 & 17.70 \\
\hline
\end{tabular}

\begin{tabular}{|c|c|c|c|c|c|c|}
\hline \multirow{3}{*}{$\begin{array}{l}\text { State/UT/India } \\
\\
\text { Andaman \& } \\
\text { Nicobar } \\
\text { Islands }\end{array}$} & \multicolumn{6}{|c|}{ No. of Public facilities } \\
\hline & \multirow{2}{*}{$\begin{array}{l}\text { PHC } \\
27\end{array}$} & \multirow{2}{*}{$\begin{array}{l}\text { CHC } \\
4\end{array}$} & \multirow[t]{2}{*}{ SDH } & \multicolumn{2}{|l|}{ DH } & \multirow{2}{*}{\begin{tabular}{|l} 
Total \\
1246
\end{tabular}} \\
\hline & & & & 3 & 34 & \\
\hline $\begin{array}{l}\text { Andhra } \\
\text { Pradesh }\end{array}$ & 1417 & 198 & 31 & 20 & 1666 & 60799 \\
\hline $\begin{array}{l}\text { Arunachal } \\
\text { Pradesh }\end{array}$ & 122 & 62 & & 15 & 199 & 2320 \\
\hline Assam & 1007 & 166 & 14 & 33 & 1220 & 19115 \\
\hline Bihar & 2007 & 63 & 33 & 43 & 2146 & 17796 \\
\hline Chandigarh & 40 & 2 & 1 & 4 & 47 & 3756 \\
\hline Chhattisgarh & 813 & 166 & 12 & 32 & 1023 & 14354 \\
\hline $\begin{array}{l}\text { Dadra \& } \\
\text { Nagar Haveli }\end{array}$ & 9 & 2 & 1 & 1 & 13 & 568 \\
\hline $\begin{array}{l}\text { Daman \& } \\
\text { Diu }\end{array}$ & 4 & 2 & & 2 & 8 & 298 \\
\hline Delhi & 534 & 25 & 9 & 47 & 615 & 20572 \\
\hline
\end{tabular}


${ }^{1}$ Dr. RK Arya, ${ }^{2}$ Dr. Urvashi Sharma, ${ }^{3}$ Dr. Arvind Dalal,

\begin{tabular}{|c|c|c|c|c|c|c|}
\hline Goa & 31 & 4 & 2 & 3 & 40 & 2666 \\
\hline Gujarat & 1770 & 385 & 44 & 37 & 2236 & 41129 \\
\hline Haryana & 500 & 131 & 24 & 28 & 683 & 13841 \\
\hline $\begin{array}{l}\text { Himachal } \\
\text { Pradesh }\end{array}$ & 516 & 79 & 61 & 15 & 671 & 8706 \\
\hline $\begin{array}{l}\text { Jammu \& } \\
\text { Kashmir }\end{array}$ & 702 & 87 & & 29 & 818 & 11342 \\
\hline Jharkhand & 343 & 179 & 13 & 23 & 558 & 7404 \\
\hline Karnataka & 2547 & 207 & 147 & 42 & 2943 & 56333 \\
\hline Kerala & 933 & 229 & 82 & 53 & 1297 & 39511 \\
\hline Lakshadweep & 4 & 3 & 2 & 1 & 10 & 250 \\
\hline $\begin{array}{l}\text { Madhya } \\
\text { Pradesh }\end{array}$ & 1420 & 324 & 72 & 51 & 1867 & 38140 \\
\hline Maharashtra & 2638 & 430 & 101 & 70 & 3239 & 68998 \\
\hline Manipur & 87 & 17 & 1 & 9 & 114 & 2562 \\
\hline Meghalaya & 138 & 29 & & 13 & 180 & 4585 \\
\hline Mizoram & 65 & 10 & 3 & 9 & 87 & 2312 \\
\hline Nagaland & 134 & 21 & & 11 & 166 & 1944 \\
\hline Odisha & 1360 & 377 & 27 & 35 & 1799 & 16497 \\
\hline Puducherry & 40 & 4 & 5 & 4 & 53 & 4462 \\
\hline Punjab & 521 & 146 & 47 & 28 & 742 & 13527 \\
\hline Rajasthan & 2463 & 579 & 64 & 33 & 3139 & 51844 \\
\hline Sikkim & 25 & 2 & 1 & 4 & 32 & 1145 \\
\hline Tamil Nadu & 1854 & 385 & 310 & 32 & 2581 & 72616 \\
\hline Telangana & 788 & 82 & 47 & 15 & 932 & 17358 \\
\hline Tripura & 114 & 22 & 12 & 9 & 157 & 4895 \\
\hline Uttar Pradesh & 3277 & 671 & & 174 & 4122 & 58310 \\
\hline
\end{tabular}




\begin{tabular}{|l|l|l|l|l|l|l|}
\hline Uttarakhand & 275 & 69 & 19 & 20 & 383 & 6660 \\
\hline West Bengal & 1374 & 406 & 70 & 55 & 1905 & 51163 \\
\hline All India & 29,899 & 5,568 & 1,255 & 1,003 & 37,725 & $7,39,024$ \\
\hline
\end{tabular}

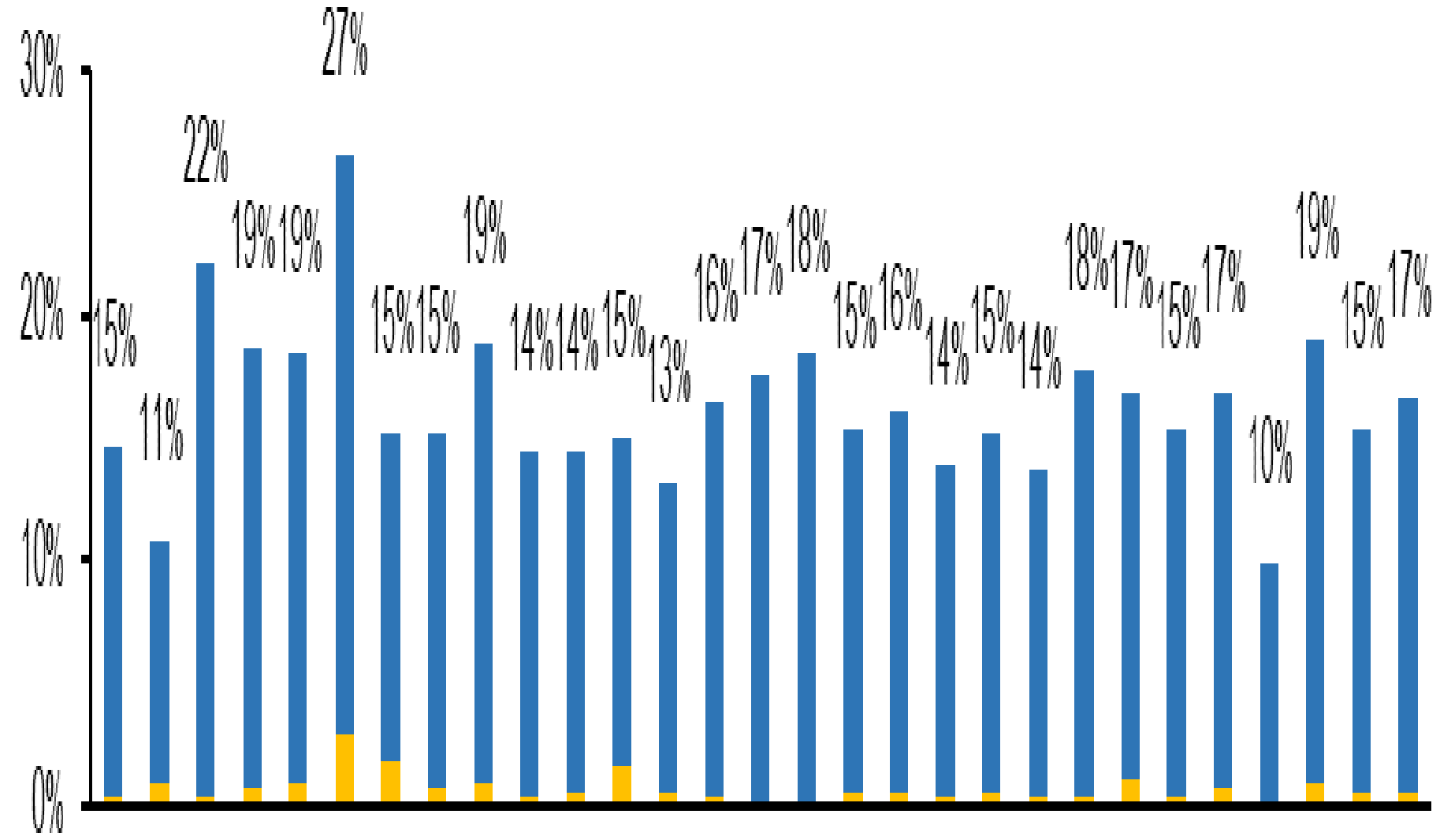

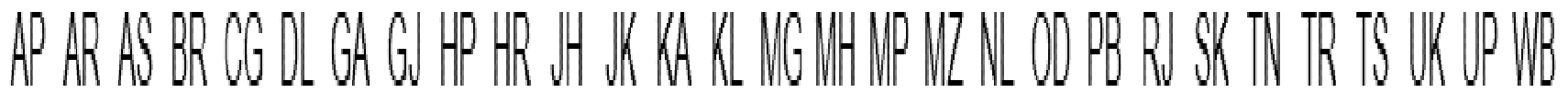

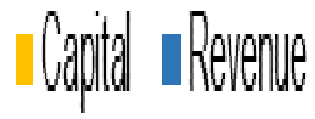

STATE WISE EDUCATION AND TRAININGWISE EDUCATION AND TRAINING 
TABLE 126: STATE-WISE CAPITAL EXPENDITURE (Concld.)

(₹ Billion)

\begin{tabular}{|c|c|c|c|c|c|c|c|c|c|}
\hline States & 2008-09 & $2009-10$ & 2010-11 & 2011-12 & 2012-13 & 2013-14 & 2014-15 & $\begin{array}{r}2015-16 \\
\text { (RE) }\end{array}$ & $\begin{array}{r}2016-17 \\
\text { (BE) }\end{array}$ \\
\hline \multicolumn{10}{|l|}{ I. Non-Special Category } \\
\hline Andhra Pradesh & 189.5 & 216.3 & 221.0 & 254.7 & 267.4 & 262.5 & 185.5 & 172.0 & 200.2 \\
\hline Bihar & 86.7 & 102.1 & 124.9 & 136.8 & 147.4 & 179.3 & 221.3 & 311.8 & 347.6 \\
\hline Chhattisgarh & 39.2 & 42.9 & 42.1 & 60.9 & 78.5 & 65.8 & 76.5 & 117.3 & 146.9 \\
\hline Goa & 10.9 & 13.0 & 14.5 & 14.7 & 12.9 & 13.5 & 16.0 & 33.5 & 39.1 \\
\hline Gujarat & 131.6 & 117.0 & 141.7 & 196.7 & 286.2 & 294.6 & 299.9 & 322.8 & 367.6 \\
\hline Haryana & 58.3 & 73.0 & 61.2 & 77.4 & 84.4 & 70.4 & 69.1 & 228.5 & 157.2 \\
\hline Jharkhand & 51.6 & 54.9 & 62.6 & 50.2 & 70.0 & 66.3 & 82.5 & 189.6 & 147.4 \\
\hline Karnataka & 123.8 & 154.3 & 179.0 & 206.4 & 203.1 & 214.6 & 250.1 & 257.7 & 331.8 \\
\hline Kerala & 43.3 & 47.0 & 61.0 & 77.4 & 85.4 & 88.8 & 81.9 & 110.8 & 147.6 \\
\hline Madhya Pradesh & 105.2 & 141.4 & 150.4 & 279.7 & 205.3 & 198.9 & 293.3 & 273.3 & 412.3 \\
\hline Maharashtra & 233.7 & 225.2 & 237.0 & 251.7 & 254.7 & 319.3 & 294.9 & 397.1 & 433.1 \\
\hline Odisha & 54.8 & 52.5 & 66.8 & 74.4 & 90.2 & 105.1 & 144.6 & 199.5 & 196.1 \\
\hline Punjab & 42.2 & 39.0 & 41.6 & 37.7 & 50.5 & 60.2 & 66.0 & 84.4 & 87.2 \\
\hline Rajasthan & 86.7 & 86.2 & 88.3 & 117.2 & 178.0 & 185.9 & 217.6 & 684.0 & 392.1 \\
\hline Tamil Nadu & 141.3 & 133.5 & 179.7 & 256.3 & 243.3 & 243.7 & 285.8 & 301.5 & 338.2 \\
\hline Telangana & - & - & - & - & - & 0.0 & 115.8 & 201.8 & 331.1 \\
\hline Uttar Pradesh & 284.3 & 317.4 & 269.1 & 298.3 & 330.0 & 425.7 & 645.8 & 948.4 & 895.8 \\
\hline West Bengal & 93.2 & 96.3 & 84.2 & 105.0 & 132.5 & 167.2 & 193.9 & 258.8 & 305.1 \\
\hline \multicolumn{10}{|l|}{ II. Special Category } \\
\hline Arunachal Pradesh & 14.0 & 15.4 & 18.3 & 22.5 & 15.7 & 20.5 & 16.8 & 37.4 & 35.3 \\
\hline Assam & 32.4 & 37.4 & 30.0 & 37.4 & 46.1 & 52.1 & 61.7 & 140.0 & 130.5 \\
\hline Himachal Pradesh & 30.5 & 28.8 & 28.9 & 34.3 & 45.4 & 39.2 & 44.2 & 52.4 & 58.5 \\
\hline Jammu and Kashmir & 63.0 & 74.2 & 71.0 & 71.8 & 66.6 & 59.3 & 65.5 & 140.9 & 195.6 \\
\hline Manipur & 17.8 & 17.1 & 20.4 & 18.6 & 16.8 & 14.5 & 16.1 & 20.0 & 19.8 \\
\hline Meghalaya & 7.5 & 6.5 & 7.6 & 11.1 & 11.2 & 12.6 & 13.8 & 19.6 & 18.1 \\
\hline Mizoram & 5.5 & 8.3 & 7.4 & 7.6 & 8.3 & 7.2 & 10.5 & 13.4 & 16.5 \\
\hline Nagaland & 10.6 & 12.7 & 13.9 & 15.7 & 15.9 & 14.8 & 13.2 & 18.9 & 19.8 \\
\hline Sikkim & 6.9 & 7.7 & 5.3 & 7.1 & 9.2 & 10.1 & 10.9 & 13.2 & 11.1 \\
\hline Tripura & 13.8 & 15.4 & 12.6 & 16.3 & 18.1 & 18.8 & 31.5 & 44.5 & 47.2 \\
\hline Uttarakhand & 24.9 & 26.4 & 23.9 & 32.6 & 52.6 & 53.1 & 59.8 & 70.1 & 73.7 \\
\hline All-States & 2003.5 & 2161.8 & 2264.3 & 2770.4 & 3025.5 & 3263.9 & 3884.9 & 5663.2 & 5902.7 \\
\hline \multicolumn{10}{|l|}{ Memo item: } \\
\hline NCT Delhi & 86.0 & 110.3 & 111.4 & 84.4 & 92.0 & 116.9 & 74.3 & 94.4 & 107.5 \\
\hline Puducherry & 3.9 & 5.0 & 5.2 & 5.3 & 5.0 & 5.7 & 8.0 & 7.0 & 7.9 \\
\hline
\end{tabular}

$\because$ Not Available. RE: Revised Estimates. BE: Budget Estimates.

Source: 1. Minus sign (-) indicates surplus in deficit indicator.

2. Figures in respect of Bihar relate to revised estimates from 1990-91 to 1994-95 and 1999-2000 to 2003-04.

3. Figures in respect of Jammu and Kashmir relate to revised estimates from 1990-91 to 1997-98 and 2001-2002 to 2009-10.

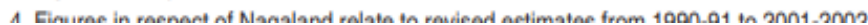


Conclusion: The differential in growth rates is clearly attributable to population stabilization efforts, stressing more on education learning outcomes through strong education system. Efforts made to create economic vibrant environment signifies that State is endeavoring to achieve self -sufficiency and sustainability. It has been observed that poverty is not dependent upon providing the transfer of funds rather it depends upon the coverage of employment generating schemes. Local resources have to be tapped to generate employment.

\section{REFERENCES}

1. https://www.timesnownews.com/education/article/international-literacy-day-2020-andhra-pradesh-worstdelhi-2nd-best-state-wise-literacy-rate-in-india/649224

2. https://en.wikipedia.org/wiki/List_of_Indian_states_and_union_territories_by_GDP

3. https://www.statista.com/statistics/271329/distribution-of-gross-domestic-product-gdp-across-economicsectors-in-india

4. https://niti.gov.in/planningcommission.gov.in/docs/data/datatable/index.php?data=datatab

5. Domestic general government health expenditure (GGHE-D) as percentage of general government $\begin{array}{lllll}\text { expenditure } & (\mathrm{GGE}) & \text { Data } & \text { by } & \text { country }\end{array}$ (http://apps.who.int/gho/data/node.main.GHEDGGHEDGGESHA2011?lang=en).

6. Current health expenditure (CHE) as percentage of gross domestic product (GDP) (\%). Data by country (http://apps.who.int/gho/data/node.main.GHEDCHEGDPSHA2011?lang=en).

7. Current health expenditure (CHE) per capita in US\$. Data by country (http://apps.who.int/gho/data/node.main.GHEDCHEpcUSSHA2011?lang=en).

8. Anand, S. (2014). Inter-state variation in public spending on higher education in India. International Journal of Humanities and Social Science Invention, 3 (9), 23 - 31.

9. Chakrabarti, A., \& Joglekar, R. (2006). Determinants of expenditure on education: An empirical analysis using state level data. Economic and Political Weekly, 41 (15), 1465 -1472.

10. Chattopadhyay, S. (2007). Exploring alternative sources of financing higher education. Economic Political Weekly, 42 (42), 4251- 4259.

11. Jana, S. K. (2012). Education in West Bengal: Looking beyond schools. New Delhi : Shipra Publications.

12. Jana, S. K. (2017). Higher education in West Bengal: An overview. Artha Beekshan, 26 (1- 2), 21 - 55.

13. Jana, S. K., Maiti, A., \& Manna, S. S. (2018). An assessment of quality of higher education using six sigma : A study in West Bengal, India. Scholars Journal of Arts, Humanities and Social Sciences, 6 (1), 259 - 266.

14. Joshi, K. M. (2006). Human capital and the economic benefits of education: Understanding the investment arguments (Working Paper, 1/06). OSED.

15. Khan, B. M. N. (2013). Public expenditure on education in Karnataka. Arthshastra Indian Journal of Economics and Research, 2 (6), 31 - 39. DOI: 10.17010/aijer/2013/v2i6/54538

16. Mallick, L., \& Dash, D. P. (2015). Does expenditure on education affect economic growth in India? Evidence from cointegration and Granger causality analysis. Theoretical and Applied Economics, XXII (4), $63-74$.

17. Ministry of Human Resource Development, Department of Secondary and Higher Education, Government of India. (2005). Report of the Central Advisory Board of Education (CABE) Committee on autonomy of higher education institutions. Retrieved from https://mhrd.gov.in/sites/upload_files/mhrd/files/documentreports/AutonomyHEI.pdf

18. Ministry of Human Resource Development, Government of India. (2007). Analysis of budgetary expenditure on education $2004-05$ to 2007 - $07 . \quad$ Retrieved from https://mhrd.gov.in/sites/upload_files/mhrd/files/statistics-new/Analysis_04-05_06-07.pdf

19. Ministry of Human Resource Development, Government of India. (2015). Analysis of budgetary $\begin{array}{lllll}\text { expenditure on 2012-13 to 2014-15. } & \text { Retrieved }\end{array}$ from https://mhrd.gov.in/sites/upload_files/mhrd/files/statistics-new/ABE2012-15.pdf

20. Ministry of Human Resource Development, Government of India. (Various Years). All India survey of higher education (AISHE) Report, Various Year. Retrieved from http://aishe.nic.in/aishe/reports;jsessionid=53F9AA1A3F7791A312461B2D321F4D07

21. Prakash, V. (2007). Trends in growth and financing of higher education in India. Economic and Political Weekly, 42(31), 3249 - 3258. doi: https://www.jstor.org/stable/4419875

22. Rajalakshmy, G. (2012). Higher education in India : Challenges and prospects. Arthshastra Indian Journal of Economics and Research, 1 (4), 25 - 32. DOI: 10.17010/aijer/2012/v1i4/54534 
23. Reserve Bank Of India. (N.D.). Database Of Indian Economy (Dbie). Retrieved From Https://Dbie.Rbi.Org.In/Dbie/Dbie.Rbi?Site=Home

24. Ode, Elijah Ojowu. "Information Literacy As A Catalyst For Socio-Economic And Political Developmentof Rural Communities In A Globally Depressed Economy." Impact: International Journal Of Computational Sciences And Information Technology (Impact: Ijcsit) 3.1 (2017): 1-8.

25. Qadri, Mohammad Ahmed, And Suwaibah Qadri. "China-Pakistan Relations: With Reference To The Political Economy." Best: International Journal Of Humanities, Arts, Medicine And Sciences (Best: Ijhams) 4.2 (2016): 187-194.

26. Brindha, N. "Current Livestock Scenario In India And Their Contribution To National Economy." International Journal Of Agricultural Science And Research (Ijasr) 7.6 (2017) 143-150

27. Qazi, S. A. Y. E. E. D. U. Z. Z. A. F. A. R., And Mohammad Saleh Miralam. "Human Resources Management Practices And Job Satisfaction In Critical Economy: An Empirical Study Of Private Banking Sector Of Saudi Arabia." International Journal Of Business Management And Research (Ijbmr), 76 (2017) 43-52

28. Choudhary, Anand. "On Way Towards A Cashless Economy Challenges And Opportunities." International Journal Of Business Management \& Research (Ijbmr) 8.2 (2018) 1-8

29. Girija, K., And M. Nandhini. "Awareness About Cash Less Economy Among Students." International Journal Of Economics, Commerce And Research (Ijecr) 8.5 (2018) 5-12 